\title{
Looking for the unseen Universe
}

\section{Physicists may soon be able to detect particles which cosmologists tell them constitute most of the Universe. But the experiments are both speculative and expensive.}

THE cooperative study of particle physics and cosmology was established as a worthwhile scientific endeavour by B. W. Lee and S. Weinberg in 1973. In an early and influential paper, they showed that simple arguments in cosmology could constrain possible values of the neutrino mass which, according to some then-current theoretical preferences might not be zero. Neutrinos are produced in the early Universe in accurately predictable numbers and, if they have mass, their contribution to the density of the Universe today can easily be calculated. But astronomers know unequivocally that visible matter in the Universe today cannot be as little as, say, one thousandth of the mass of the unknown invisible component, which sets an upper limit for the neutrino mass.

This illustrates the general style of argument in cosmology and particle physics, although the chains of reasoning have become more intricate and more hedged about. For example, if a neutrino of a particular mass is ruled out by Lee and Weinberg's calculations, then it can be saved by assuming it is unstable, and decays into massless particles. But then one must worry about what the decay products do to standard cosmological evolution. They may heat the Universe or distort the microwave background or upset the synthesis of helium, deuterium and lithium in the first three minutes of the Big Bang.

If cosmologists are ingenious at thinking up arguments to constrain what elementary particles can do, particle physicists are just as ingenious at evading the constraints. The neutrino, for example, can be made to decay into something invisible and innocuous whose presence the most assiduous of cosmologists cannot detect. If there was a single theme that emerged from a recent gathering of astronomers and physicists*, it was the need for hard facts, from cosmology or experimental physics, to set boundaries on the imagination of theorists. Massive neutrinos were mentioned often, and enlisted in a variety of causes, but what mass they have, if any, is little better known now than in 1973 .

Over the years, the relative roles of particle physics and cosmology have changed. At first, simple cosmological models were used to test and provide limits on exotic theories of particle physics. But cosmologists have begun to invent more sophisticated evolutionary models dependent on particles which, it is hoped, physicists will one day be able to supply. This has given participants on both sides of the enterprise a bad name.

There is still no satisfactory theory of the formation of galaxies. Observers have catalogued galaxy positions and redshifts in increasing numbers, but their distribution in space still defies accurate description. Galaxies congregate on filaments and knots, and there are large regions of space devoid of them. But this empirical picture has not been made quantitative. And there is no consensus on whether the empty regions - the voids - are empty of matter or only of matter in luminous form. In short, astronomers cannot say how visible matter is distributed in the Universe and do not know whether the invisible matter is distributed in the same way as galaxies, or more uniformly.

This leaves cosmologists with some difficult problems to solve and a good deal of latitude in which to solve them, which encourages speculation. The most serious obstacle that galaxy formation theories must negotiate is the absence of measurable temperature fluctuations in the microwave background. The Universe at 100,000 years of age have been remarkably free from irregularities, but now, when it is ten thousand million years old, there are superclusters and voids. Finding ways for these enormous structures to grow from almost nothing in the permitted interval is difficult; cosmologists suppose that the chief agent is whatever material constitutes the dark matter, and that the visible and familiar stuff of galaxies merely falls into place later.

A trap into which cosmologists can fall is to develop a theory around some putative elementary particle, whose properties they particularly like, to describe a largescale distribution of matter so as to explain the presence and arrangement of galaxies which make up a dynamically insignificant fraction of the modern Universe.

There is now a range of theories of galaxy formation sharing some uncontroversial elements but whose finer differences cannot be resolved for lack of data. The good news in Bologna was twofold: cosmologists are anxious to find independent tests of their ideas, and physicists have some hope of providing them.

Massive neutrinos have been supplanted as the most popular dark matter candidates by axions. (Neutrinos cannot explain the small-scale structure of galaxy cluster- ing.) Axions, a concomitant of the PecceiQuinn explanation of CP conservation in strong interactions, are much less massive but much more numerous than cosmological neutrinos, and have lower velocities. These persuade cosmologists that it has a much better chance of playing the dynamical role required of dark matter constituents. The greatest drawback of the axion, though, is that there is not one piece of experimental evidence that it exists.

An experiment now in progress at Brookhaven National Laboratory, New York, may provide that evidence, or discount the axion as a dark matter candidate. In the presence of virtual photons provided by a strong magnetic field, axions can convert into microwave photons at a potentially measurable rate, if indeed we are bathed in a sea of cosmologial axions.

A tunable microwave cavity inside a large superconducting magnet thus becomes a cosmological axion detector. The frequency of the detectable microwave photons is directly proportional to the axion's mass, so by tuning the cavity, axions in a likely mass range can be sought out. There is a variety of other constraints on the axion mass, from laboratory experiments as well as from astrophysics, making a mass from $10^{-3}$ to $10^{-5} \mathrm{eV}$ the only possibility.

Unfortunately, the sensitivity of the experiment is about an order of magnitude less than what is needed, and the lack of detection so far is as expected. But the sensitivity of the detector increases with the volume and the square of the magnetic field, and it seems reasonable to imagine that a thousandfold increase could be obtained. This would allow detection or dismissal of the axion hypothesis with equal firmness.

But the problem now, in blunt terms, is to decide how much it is worth spending to test one admittedly speculative theory of the structure of the Universe. The present experiment used an old magnet built some years ago at Brookhaven, but a bigger detector would require a custom-built and consequently expensive device. And those who spent a couple of years designing and performing this prototype experiment may prefer not to devote the many years needed for the bigger version.

David Lindley

* Third ESO-CERN meeting on astronomy, cosmology and fundamental physics, 16-20 May, Bologna. 